\title{
Atuação da comissão de farmácia e terapêutica em um hospital de ensino
}

\section{Performance of the pharmacy and therapeutics committee in a teaching hospital}

\author{
Lilian P. Primo', Elias de Carvalho¹, Adriano E. U. de Lima¹, Claudia B. T. Gandolfi', Kátia M. Padula', \\ Luís F. B. Bózoli', Alexandra C. Abramovícius², Maria Eulália L. V. Dallora ${ }^{3}$, Antonio Pazin Filho ${ }^{4}$
}

\begin{abstract}
RESUMO
Introdução: As atividades hospitalares caracterizam-se por um acentuado dinamismo em consequência do surgimento de novas tecnologias em saúde, tais como medicamentos. Uma unidade hospitalar, devido suas características de ensino, pesquisa e atendimentos de alta complexidade, possui maior concentração de diferentes tipos de tecnologias em saúde. O Hospital das Clínicas da Faculdade de Medicina de Ribeirão Preto -USP (HCFMRP-USP) é uma instituição hospitalar, de qualidade comprovada, inserido no SUS como referência terciária/quaternária e que conta com a Divisão de Assistência Farmacêutica (DAF) para desenvolvimento das ações de atenção a saúde. A DAF para auxílio da gestão de recursos, seleção e padronização de medicamentos adotou como estratégia a Comissão de Farmácia e Terapêutica (CFT). A CFT é uma instância colegiada, de caráter consultivo e deliberativo, estabelecida pela Organização Mundial de Saúde como ferramenta de estratégia para monitorar e promover a qualidade no uso do medicamento, porém estudos que sobre a atuação das CFTs no Brasil são incipientes. Desta forma, este estudo pretende apresentar a CFT do HCFMRP-USP. Objetivos: apresentar a composição, atribuições e metodologia de trabalho da CFT, bem como desenvolver uma análise crítica de seu atual funcionamento. Metodologia: Foi realizado estudo descritivo e exploratório com o objetivo de descrever o atual funcionamento da CFT do HCFMRP-USP. Foram buscadas portarias, regulamentações internas e foi realizada revisão bibliográfica sobre a CFT. Para Análise crítica do atual funcionamento, foi selecionado dentre os itens padronizados pela comissão aquele que se enquadrasse como pertencente a classificação $\mathrm{A}$ e $\mathrm{V}$, após o cruzamento das curvas ABC e VEN, cujo item selecionado foi o medicamento Sevoflurano. Resultados: ACFT foi instituída no ano de 2010 em substituição a extinta comissão de padronização. Desde então, a CFT analisou 134 solicitações e destas 41 foram padronizadas. O medicamento sevoflurano foi incorporado no HCFMRP-USP em 2010 e, a partir deste ano, observa-se um aumento gradativo do consumo do mesmo. Entretanto, após análise das prescrições do referido medicamento no ano de 2012, foi observado que a dispensação do sevoflurano não segue as especificações do perfil de pacientes conforme estabelecido no protocolo instituído no momento da padronização. Conclusões: Portanto, concluímos que a implantação da CFT foi uma estratégia que proporcionou a padronização racional. Entretanto, observa-se que não há controle da dispensação e utilização do medicamento de acordo com o protocolo estabelecido no momento da padronização. Salientamos que o controle do uso do Sevoflurano não é atribuição da CFT devendo esta atribuição ser delegada à área técnica responsável.
\end{abstract}

Palavras-Chave: Comitê de Farmácia e Terapêutica; Medicamentos Essenciais.

1. Alunos de Pós-Graduação - Mestrado Profissionalizante Gestão em Organizações de Saúde.

2. Diretora da Farmácia do HCFMRP-USP.

3. Assistente Administrativa do HCFMRP-USP e Orientadora do Programa de Pós-Graduação - Mestrado Profissionalizante Gestão em Organizações de Saúde

4. Professor Associado do Departamento de Clínica Médica da FMRP-USP e Orientador do Programa de Pós-Graduação - Mestrado Profissionalizante Gestão em Organizações de Saúde.
Correspondência: Divisão de Assistência Farmacêutica - HCFMRP-USP Avenida dos Bandeirantes, n. ${ }^{\circ}$ 3900, Campus Universitário, Monte Alegre - Ribeirão Preto, São Paulo. CEP 14048-900

Artigo recebido em 16/06/2014 Aprovado para publicação em 14/08/2014 


\begin{abstract}
Introduction: The hospital activities are characterized by a highly dynamism as a result of new health technologies such as medicines. A hospital due its characteristics of teaching, research and high complexity care, has the highest concentration of different types of health technologies. The Hospital das Clínicas da Faculdade de Medicina de Ribeirão Preto - USP (HCFMRP-USP) is a hospital, with proven quality, inserted in the SUS as a tertiary/quaternary referral and has the Pharmaceutical Services Division (DAF) for development of actions of health care. To aid resource management, selection and standardizationof drugs, DAF adopted the strategy of Pharmacy and Therapeutics Committee (CFT). The CFT is a collegial, consultative and deliberative body, established by the World Health Organization as a strategy tool to monitor and promote the quality in the use of medicine, but studies of CFTs are incipient in Brazil. Thus, this study aims to present the CFT of HCFMRP-USP. Objectives: To introduce the composition, responsibilities and working methods of CFT, as well as a critical analysis of its current operation. Methods: A descriptive study aimed to describe the current functioning of the CFT of HCFMRP-USP was performed. Ordinances, internal regulations were surveyed and a bibliographic review of the CFT was performed. To the critical analysis of the current operating, was selected by the committee from the standard one that would fit classification as belonging to "A" and "V" items after the crossing of the curves ABC and VEN, whose selected item was the medicine Sevoflurane. Results: The CFT was established in 2010 to replace the defunct standardization committee. Since then, the CFT examined 134 requests and 41 of these were standardized. The Sevoflurane drug was incorporated into the HCFMRP-USP in 2010 and, starting that year, there was a gradual increase in the consumption of the same. However, after analyzing the requirements of the drug in 2012, it was observed that the dispensation of Sevoflurane does not follow the specifications of the patient profile as established in the protocol established at the time of standardization. Conclusions: We concluded that the implementation of CFT was a strategy that provided a rational standardization. However, it is observed that there is no control of dispensing and use of the product according to the protocol established at the time of standardization. We emphasize that control the use of Sevoflurane is not responsibility of the CFT and this assignment should be delegated to the responsible technical area.
\end{abstract}

Key words: Pharmacy and Therapeutics Commitee; Drug, essential.

\section{Introdução}

As atividades hospitalares caracterizam-se por um acentuado dinamismo em consequência do surgimento de novas tecnologias em saúde, tais como medicamentos. A incorporação dos medicamentos à lista de padronização remete a necessidade de uma seleção cuidadosa, buscando eficácia, segurança e qualidade dos fármacos, além da avaliação impacto econômico da aquisição dos mesmos. ${ }^{1,2}$

Uma unidade hospitalar, devido a sua característica de ensino, pesquisa e de atendimentos de alta complexidade, possui maior concentração de diferentes tecnologias. Desta forma, estas unidades devem adotar estratégias de entrada e saída de medicamentos, de forma a manter uma padronização atualizada e que promova o seu uso racional. ${ }^{1,3}$

O Hospital das Clínicas da Faculdade de Medicina de Ribeirão Preto - USP é uma Instituição Hospitalar de qualidade comprovada, inserido no SUS como referência terciária/quaternária e que abrange uma população de cerca de quatro milhões de habitantes.
Para desenvolvimento das ações de atenção à saúde, conta com a Divisão de Assistência Farmacêutica (DAF) que é a unidade responsável por desenvolver as atividades ligadas à seleção, aquisição, armazenamento, distribuição e dispensação de medicamentos. Esta unidade tem como diretrizes: promover o uso racional de medicamentos assim como contribuir para a qualidade da assistência prestada ao paciente, buscando a melhoria contínua em todos os processos.

O orçamento de custeio da DAF representa cerca $30 \%$ de todo o orçamento do Hospital, cuja média mensal é de R $\$ 3$ milhões de reais, totalizando R\$ 36 milhões/ano. Entretanto, para a gestão destes recursos é importante o uso de ferramentas que possam orientar a tomada de decisão de quais medicamentos e insumos deverão fazer parte da padronização da instituição. Assim, uma das estratégias adotadas para seleção, padronização e alocação de recursos para a aquisição foi a criação de uma Comissão de Farmácia e Terapêutica (CFT).

A CFT é uma instância colegiada, de caráter consultivo e deliberativo, e seu estabelecimento ajuda no processo de assistência à saúde, no esforço para 
conter o aumento da resistência antimicrobiana, adequar o uso dos medicamentos de forma a reduzir o número de reações adversas a medicamentos (RAM), devido a possíveis erros no uso destes, e ainda, o uso daqueles relativamente perigosos. A CFT pode ser classificada, ainda, como um recurso fundamental na busca pela eficiência na utilização dos recursos em saúde. . $^{2,3,4,5}$

A Organização Mundial da Saúde estabelece a CFT como uma das estratégias para monitorar e promover a qualidade no uso dos medicamentos. Estudos mostram que em países desenvolvidos como Canadá e Estados Unidos as CFTs possuem papel fundamental nas variáveis econômicas, sociais, sanitárias para avaliar a incorporação de medicamentos a padronização das unidades hospitalares. ${ }^{2,5}$

No Brasil, a atuação das CFTs é incipiente e existem poucos estudos sobre a sua existência e a metodologia de trabalho utilizada. ${ }^{1,5}$

Portanto, o presente estudo pretende apresentar a CFT do HCFMRP-USP quanto a sua funcionalidade e desenvolver uma análise crítica do seu atual funcionamento.

\section{Objetivos}

- Apresentar a composição, atribuições e metodologia de trabalho da CFT do HCFMRP-USP.

- Desenvolver a análise crítica do funcionamento atual da Comissão e propor sugestões de melhorias para os processos de trabalho.

\section{Métodos}

Foi realizado estudo descritivo e exploratório da composição, atribuições e metodologia de traba- lho da Comissão de Farmácia e Terapêutica (CFT). O estudo foi realizado no Hospital das Clínicas da Faculdade de Medicina de Ribeirão Preto - USP (HCFMRP-USP) no período de agosto a dezembro de 2013.

Para conhecermos com detalhes o funcionamento da CFT no HCFMRP-USP, buscamos portarias e regulamentações internas, além de dados relativos à sua atuação. Foi realizada revisão bibliográfica e foram analisados todos os documentos relativos à seleção de medicamentos pela CFT.

Para seleção e análise crítica do funcionamento atual da Comissão foi selecionado um item padronizado pela Comissão por meio de análise do cruzamento das Curvas ABC e VEN. Este cruzamento de curvas foi instituído pelo HCFMRP-USP a fim de separar os itens de maior importância ou impacto financeiro (Curva $\mathrm{ABC}$ ) e segundo sua especialidade técnica (curva VEN) ${ }^{2}$ Desta forma, a curva ABC designa os medicamentos como: os da classe A correspondentes aos de maior valor, os de classe $\mathrm{B}$ de valor intermediário e os de classe $\mathrm{C}$ correspondem aos medicamentos com menor impacto financeiro. Já a curva VEN classifica da seguinte maneira: classe $V$ sendo os medicamentos vitais, classe $\mathrm{E}$ os essenciais e os da classe $\mathrm{N}$ os não essenciais.

O critério escolhido para resultado e discussão neste estudo foi o medicamento padronizado pela CFT que se enquadrasse na classificação A e V, sendo selecionado o medicamento sevoflurano. Com a decisão de focar especificamente no medicamento acima, pesquisamos informações mais detalhadas nos Sistemas de Prescrição Eletrônica e Gestão de Materiais do HCFMRP-USP. Através deles foi possível verificar todas as prescrições e dispensações de sevoflurano realizadas no ano de 2012.

Tabela 1: Exemplo de medicamentos padronizados pela Comissão de Farmácia e Terapêutica por meio do cruzamento das curvas ABC e VEN no HCFMRP-USP

\begin{tabular}{ccl}
\hline Curva ABC & Classe VEN & Medicamento \\
\hline A & V & Sevoflurano $100 \%$, inalatório, frasco $250 \mathrm{ml}$ \\
B & V & Azacitidina injetável, frasco-ampola 100mg \\
B & V & Dextrocetamina, cloridrato injetável Fr-amp $500 \mathrm{mg} 10 \mathrm{ml}$ \\
B & V & Insulina de ação rápida 100 ui/ml frasco \\
B & V & Mitotane comprimido $500 \mathrm{mg}$ \\
B & V & Toxina botulínica injetável frasco 100ui \\
B & V & Caspofungina injetável frasco-ampola $50 \mathrm{mg}$ \\
\hline
\end{tabular}




\section{Resultados e Discussão}

\section{A composição, atribuições e metodologia de trabalho da CFT}

No HCFMRP-USP a CFT foi instituída no ano de 2010 em substituição a extinta Comissão de Padronização. Desde então, trabalha em conjunto com a DAF e outras instâncias de forma a otimizar o processo de padronização e uso racional do medicamento, assim como ferramenta na gestão dos recursos a serem utilizados.

A composição da CFT possui característica multiprofissional, sendo composta por representantes com autonomia de decisão das seguintes áreas: Diretoria Clínica; Administração; Divisão de Assistência Farmacêutica; Divisão de Enfermagem; Comissão de Controle de Infecção Hospitalar (CCIH) e Especialidades Médicas. ACFT pode contar, ainda, com assessores "ad hoc", que são profissionais pertencentes ou não a Instituição, com a finalidade de fornecer mais subsídios para emissão de parecer técnico e tomada de decisão. ${ }^{6}$

Cabe a CFT a elaboração e implementação de intervenções para melhorar o uso dos medicamentos, devendo a direção do hospital delegar autoridade e apoiar as atividades executadas e as decisões tomadas pela mesma. Dentre suas atribuições realiza ações de assessoramento farmacoterapêutico, investigação cientifica, educação permanente, seleção e padronização de medicamentos e insumos.

Como metodologia de trabalho a CFT realiza análise detalhada da nova alternativa terapêutica. Esta análise é baseada em estudos de revisão sistemática e metanálise relacionados ao produto selecionado. Este método tem por objetivo oferecer resultados de eficácia e segurança dos medicamentos, e confirmar as informações inerentes ao novo produto, o conhecimento da real dimensão dos benefícios dessa nova tecnologia e subsidiar a tomada de decisão da incorporação ao serviço de saúde de forma científica e custo-efetivo. Por meio deste método, também é possível garantir a racionalidade da terapia medicamentosa a ser implementada, oferecendo o meio para controle e gerenciamento da utilização dos mesmos. A CFT analisa, ainda, o impacto orçamentário para incorporação desta nova tecnologia, pois os recursos financeiros para aquisição deste produto farão parte da alínea anual de recursos destinados à aquisição de medicamentos e, portanto, será de responsabilidade dos gestores da instituição. Após a decisão pela padronização, são realizados protocolos de tratamentos baseados na clínica, posologia e frequência de uso do medicamento.

As solicitações de incorporação de medicamentos antimicrobianos (antibacterianos, antifúngicos e antivirais) são encaminhadas pela CFT à Comissão de Uso e Controle de Antimicrobianos (CUCA) do HCFMRP USP, afim de que esta avalie em termos econômicos e desfechos clínicos, a viabilidade de sua padronização. Em algumas situações, determinadas em reunião com seus membros, a CUCA opta por utilizar o produto em avaliação em casos clínicos específicos, antes de emitir seu parecer final favorável ou não. A referida Comissão acredita ser importante basear seu parecer após obtenção de dados através de estudos locais e com pacientes provenientes do SUS devido suas características socioculturais.

Após aproximadamente 03 anos de funcionamento a CFT analisou 134 solicitações de padronização de medicamentos. Destes, 41 foram padronizados e elaborados protocolos de tratamentos.

\section{Análise crítica do funcionamento atual da Comissão}

O medicamento escolhido, para avaliar criticamente as decisões da CFT foi o sevoflurano, por se enquadrar nas curvas A e V, ou seja, é um medicamento considerado vital para a instituição e de grande impacto financeiro no custeio hospitalar. O sevoflurano foi padronizado no ano de 2010 e desde então, observa-se um aumento gradativo no consumo deste anestésico, mesmo tendo outros padronizados com ação farmacológica similar, como o isoflurano.

Segundo o Protocolo apresentando à CFT para padronização e utilização do sevoflurano, o perfil dos pacientes que deveriam receber este anestésico seriam crianças de 1 a 6 anos e pacientes maiores de 60 anos de idade, e a dosagem utilizada deveria ser individualizada e titulada para o efeito desejado de acordo com a idade e quadro clínico do paciente.

Dados retirados do sistema de Prescrição Eletrônica evidenciaram no ano de 2012, prescrições para 867 pacientes, sendo para cada um, prescrito um volume correspondente ao de um frasco. Ao analisarmos as prescrições do medicamento a farmácia, identificamos que o sevoflurano esta sendo prescrito e dispensado para outros perfis de pacientes que não os indicados no protocolo estabelecido. Desta forma, 


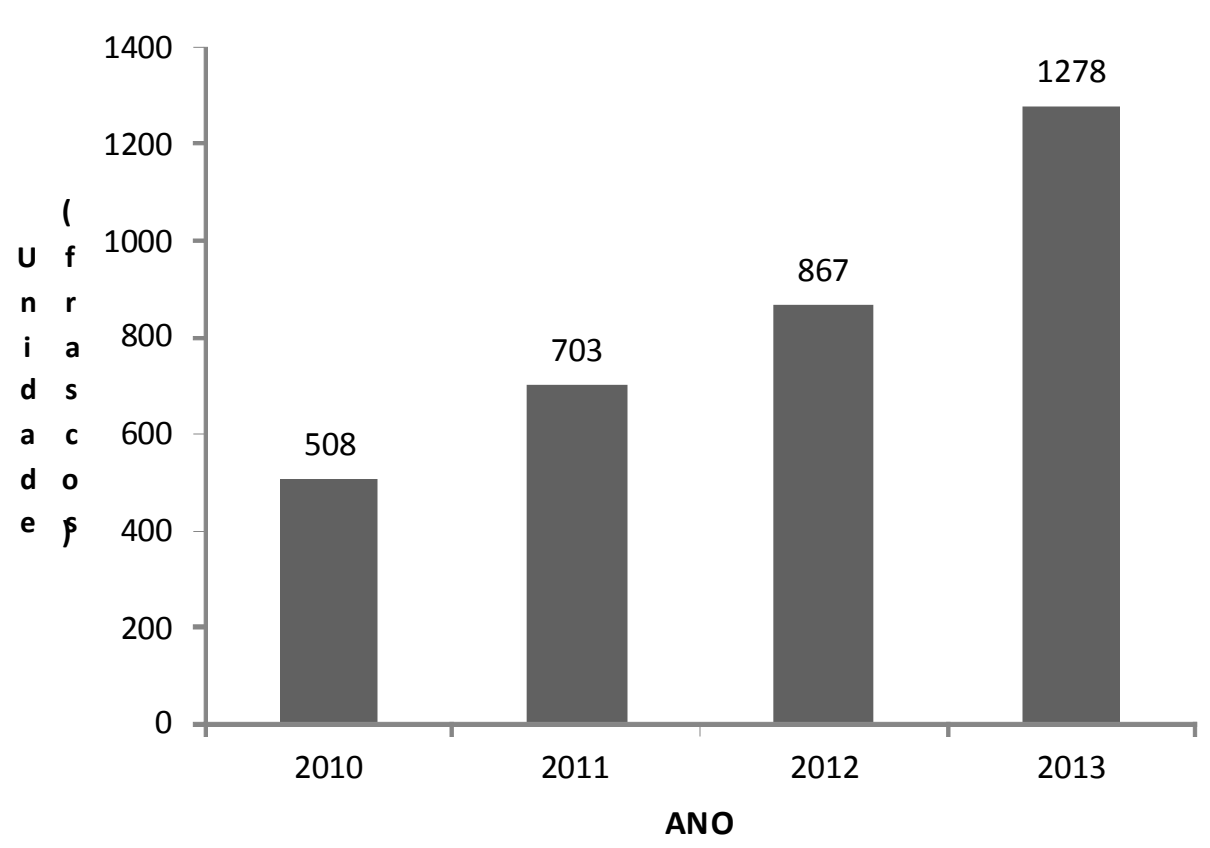

Gráfico 1: Consumo do Sevoflurano nos anos subsequentes a sua padronização.

observamos que, no caso apresentado, não foram realizadas revisões periódicas do protocolo estabelecido durante a padronização, o que pode induzir a discrepâncias entre o que foi utilizado e o referido protocolo. Além disso, após confrontarmos a prescrição com a evolução anestésica, observamos que o volume de medicamento utilizado em cada paciente não era o correspondente ao volume de um frasco, restando desta forma medicamento, não sendo possível rastrear o destino final. Esta questão também nos aponta que o Sistema de Prescrição Eletrônica tem limitações que devem ser corrigidas, pois no caso deste anestésico a quantidade informada corresponde à necessidade de requisição à Farmácia e não à prescrição para o paciente.

\section{Propostas de melhorias para os processos de trabalho}

Por décadas, o uso de medicamentos inadequados para tratar doenças vem levando ao agravamento de problemas relacionados ao cuidado à saúde, resultando no aumento da morbidade, mortalidade, custos e, mais recentemente, o surgimento de resistência antimicrobiana $^{2,7}$. Como instâncias dentro de hospitais, as CFTs são as responsáveis pela avaliação do uso clínico dos medicamentos, desenvolvendo políti- cas para gerenciar o uso, a administração e o sistema de seleção. Como um foro, cria espaço para avaliar e discutir todos os aspectos do tratamento medicamentoso, orientando os departamentos médicos, de enfermagem, administrativos e de farmácia sobre temas relacionados a medicamentos. Estas funções são de extrema importância para a melhoria da gestão, mas no caso do estudo ainda não ocorre de fato. ${ }^{2,5}$

Visando buscar ferramentas que minimizem e eliminem as discrepâncias entre as indicações do protocolo e a dispensação do medicamento, as seguintes propostas foram elaboradas:

- Atrelar os critérios dos protocolos clínicos com o Sistema de Prescrição Eletrônica, de modo que seja dispensado somente conforme indicação do protocolo, iniciando pelo medicamento Sevoflurano;

- Divulgação do protocolo no Ambulatório Pré-Anestésico cujo medicamento seja prescrito conforme indicação de uso.

- Implantação de farmácia satélite no Bloco Cirúrgico para gerenciamento da dispensação dos medicamentos.

Cabe ressaltar as implicações da importância no cumprimento destas ações para a gestão, para a clínica e também para a formação médica e da equipe multidisciplinar no atendimento Hospitalar. 


\section{Conclusões}

A implantação da CFT foi uma estratégia que proporcionou a padronização racional e baseada em evidências científicas de qualidade, somada ao perfil de utilização e de ponderação quanto aos gastos envolvidos, estabelecendo-se como importante instrumento para melhorar a tomada de decisão para a seleção de medicamentos. Porém, com base na análise do medicamento Sevoflurano, concluímos que o controle do uso do medicamento, bem como a sua utilização podem não estar sendo seguidas conforme o protocolo clínico estabelecido. Salientamos que o controle da utilização e dispensação não são atribuições da CFT, devendo esta atribuição ser delegada especificamente a área técnica que deveria dispor de mecanismos para fazer cumprir as deliberações da CFT.

\section{Referências}

1. Lima ECL, Sandes VS, Caetano R, Osorio-de-Castro CGS.. Incorporação e gasto com medicamentos de relevância financeira em hospital universitário de alta complexidade. Cad Saúde Colet., (Rio J.). 2010; 18: 551-9.

2. Magarinos-Torres R, Pagnoncelli D, Cruz-Filho AD, Osorio-deCastro CGS. Vivenciando a Seleção de Medicamentos em Hospital de Ensino. Rev Bras Educ Méd. 2010; 35: 77-85.

3. Trindade EA. A incorporação de novas tecnologias nos serviços de saúde: o desafio da análise dos fatores em jogo. Cad Saúde Pública. 2008; 24: 951-64.

4. Cipriano SL, Moreira RPP, Cunha GWB, Sforsin ACP, Pinto VB. Comissão de Farmácia e Terapêutica. Pharmacia Brasileira $\mathrm{n}$ 우. SBRAFH, 2011.

5. Marques DC, Zucchi P. Comissões Farmacoterapêuticas no Brasil: aquém das diretrizes internacionais. Rev panam salud pública. 2006; 19: 58-63.

6. BRASIL. Ministério da Saúde. Secretaria de Atenção à Saúde. Departamento de Atenção Especializada. Protocolos clínicos e diretrizes terapêuticas: v. 2 - Brasília, 2010.

7. BRASIL, Ministério da Saúde. Portal da saúde. Protocolos Clínicos e Diretrizes Terapêuticas. Disponível em: http:// portalsaude.saude.gov.br/portalsaude/index.cfm? portal=pagina . visualizarTexto\&cod Conteudo $=5931 \& \operatorname{cod}$ ModuloArea $=659 \&$ chamada=protocolos-clinicos-e-diretrizesterapeuticas. [Acesso em 28 de setembro de 2013]. 